\title{
How To Improve The Ethical Consideration Of Acupuncture Clinical And Basic Research
}

\author{
Hui Hui Tan ${ }^{1}$, Yun Jin Kim² \\ ${ }^{1}$ School of Traditional Chinese Medicine, Xiamen University Malaysia. Jalan Sunsuria, Bandar \\ Sunsuria, Sepang, Selangor, 43900, Malaysia \\ ${ }^{2}$ School of Traditional Chinese Medicine, Xiamen University Malaysia. Jalan Sunsuria, Bandar \\ Sunsuria, Sepang, Selangor, 43900, Malaysia \\ Corresponding Author: Yun Jin Kim \\ E-mail: yjkim@xmu.edu.my
}

\begin{abstract}
In this current perspective the aim is the ethical consideration of acupuncture in clinical research. The introduction of acupuncture and its benefit in human health was briefly stated, also gave an overview of the method of carrying out acupuncture clinical research and the choice of control procedure, while the problems faced by researchers regarding the research such as the difficulty of blindness was also put into consideration. Introduced the importance of clinical research in order to prove the medical effect of acupuncture which then makes the acupuncture intervention being more widely accepted in medical treatment. Furthermore, brings up the method to perform ethical research of acupuncture which can be the guidelines in the study of acupuncture clinical research. The seven requirements for ethical research that must be abide were explained in this review. Even though acupuncture had become a common intervention in the medical field, however, more afford was required to solve the acupuncture study-related ethical issues.
\end{abstract}

Keywords: Ethics, Acupuncture, Clinical research

\section{Introduction}

"Acupuncture" is originated from the Latin word 'Acus' means needle, while 'puncture' gives the meaning of dip or punch. Thus, acupuncture gives the meaning of the sense of needle immersion [1]. Acupuncture is known as a therapeutic procedure that stimulates acupoints on the body intending to obtain therapeutic effects. It can be done by using a needle, vacuum, pressure, ultrasound, laser, or electric current [1]. Acupuncture has become a popular Complementary and Alternative Medicine (CAM) in many countries. According to a bibliometric analysis done by $\mathrm{Ma}$ et al [2], there are around 13,320 acupuncture-related publications were identified from 1995 to 2014. Besides, there is a striking trend noticed in which the proportion of randomized clinical trials (RCTs) has been increased from $7.4 \%$ in 1995 to $20.3 \%$ in 2014 . The growth overstepping the 4.5 $\%$ proportional growth of RCIs in the field of biomedicine. Acupuncture therapy is endorsed by roughly $25 \%$ of general practitioners (GPs) in the United Kingdom while it is mostly used to cure chronic pains. In 2013, there is reported that acupuncture was utilized in 183 countries according to a survey by the World Federation of Acupuncture-Moxibustion Societies [3].

The authors will discuss the perspective viewpoint available to perform clinical research and the problems that be found in conducting the research. Besides, also pays attention to the ethical issues 
that have to be considered in research and the way to conduct ethical research in respective to acupuncture studies.

\section{Carrying Out Acupuncture Clinical Research and the Problem Faced}

Randomized controlled trials (RCTs) were regarded as the "gold standard" for demonstrating the effectiveness of healthcare interventions among all of the controlled studies. A standard RCT has three main characteristics: randomization, control, and blind or hidden. Allocation concealment and good randomness ensure the fairness and the comparability between subjects in the experimental group and the control group, as well as to overcome the selection bias problem. Effective controls can control for placebo and/or nonspecific effects and reveal the "real" therapeutic effect. Blindness can ensure the biases caused by the patient's expectations and preferences are minimized. However, due to acupuncture is a complex non-pharmacological intervention, it is "made up of several interrelated components", thus, designing an RCT that maintains the scientific accuracy of the trial design while balancing the nature of acupuncture has been a challenge. Regarding this, the WHO issued the "Guidelines for Clinical Research on Acupuncture" which described the basic elements of clinical investigation on acupuncture and moxibustion, such as patient selection, blinding technique, study site, and control design [4].

The choice of control procedure has become a research question. It depends on the aim of the study. If the study aims to test whether acupuncture shows improvement in the patient than doing nothing for the condition, then the control group does not need to receive anything. The acupuncture could be credible if only the acupuncture be proved to be beneficial than placebo. While, if the research question is testing whether acupuncture provides a better therapeutic effect than the current treatment, then the control group will has to receive the current treatment. For the research test for whether acupuncture has any specific effect, there will be a procedure that is indistinguishable from the real acupuncture intervention. Controlled trials will need the involvement of 'placebo' which can be known as an inert treatment that induces perception in participants that the intervention and placebo is unable to be distinguished from the real treatment. However, placebo control is arguing to be unethical as the patient would not receive the real treatment [5]. Besides, the effect of penetration of needle on the skin might induce treatment effect of acupuncture which implies that the placebo effect not always be regarded as inactive [6-7]. Thus, utilize the term 'sham' acupuncture to bring the meaning of needling to inappropriate points and using minimally acupuncture technique which implies needling superficially [8]. There is noninsertion sham control available which is similar to the actual acupuncture process, but the needles do not penetrate the subject's skin, for example, air guide tubes, toothpicks, semi-blunt needles, and non-penetrating needle devices. This technique aims to control the psychological effects of acupuncture. However, it is impossible to perform on subjects who have acupuncture experience before as they may be able to differentiate the real acupuncture from sham controls. Researchers could recruit subjects with little or no acupuncture experience. The disadvantage of non-inserted sham controls is that the blinding effects may not last long, as subjects may learn real acupuncture experiences through visits to other acupuncturists, or due to the therapeutic effects, they experience in long-term research studies. For example, one study showed that subjects were successfully blinded at week 4, but it failed at week 26 [3].

Besides, appropriate blinding minimizes performance bias, confirmation bias, and detection bias. Acupuncture treatment involves not only the instruments but also the process and techniques of acupuncture, such as needle insertion and manipulation. This makes it impossible for doctors to go blind, thus most acupuncture trials are single-blind, meaning patients are blind. To reduce subject identification bias, interventions should be carefully designed to effectively blind subjects. For example, a blind clip or a screen can be used to block a subject's view while an acupuncturist conducts real or fake acupuncture in the lower extremities of the patient. As the subject's expectations and the subject-acupuncturist relationship may cause potential bias, the acupuncturist is better to communicate with the patient in a neutral/concise manner during research and avoid communication about their trial experience to remain blinding. Results evaluators should keep blind to reduce the test bias, and statisticians also keep blind to ensure the data be analyzed and interpreted without bias. 
For the treatment that is proven effective in placebo controlled RCTs, it can be introduced into medical practice. After undergoing further research, if the new treatment is proven to be effective than a standard treatment, it can supplant that standard treatment. However, for treatment that is no better than placebo, will be abandoned as the treatment that proves to be no better than placebo has no clear therapeutic effect that can take precedence over the risks of harm [9].

\section{Ethical Concern of Clinical Research}

Historically, doctors have been in a privileged position to conduct research. This caused some researchers to conduct unethical research. The Helsinki Declaration was born to inform biomedical researchers of the principles of clinical research. The Declaration emphasized the principles of good clinical practice, including respect for human dignity and the principle of justice. Research should not take precedence over the health, well-being, and care of the subjects [10].

Ethical concerns take place in the four basic principles of research which are weighting of riskbenefit, respect of human beings, merit and integrity, and justice. These principles lay the foundation for any argument regarding human research ethics. It also can be applied in all research areas as well as acupuncture research and Chinese Herbal Medicine research [11]. Ethical norms act as the goals of research and must be obeyed by researchers. Ethical norms are important to make sure that the aim of the study which is expanding of truth and honest knowledge can be achieved since ethical norms strictly prohibit the behavior of falsifying, fabricating, and misrepresenting the study result. Besides, ethical norms ensure the moral value such as trust, mutual respect, and accountability was carried out by the researchers. This is much more important in the collaboration between the researchers to protect researchers' ideas and experimental results. Ethical norms are crucial to protect intellectual property work and promote the researchers held accountable to the public. Making sure researchers take consideration of human rights, animal welfare, and social responsibilities as it is in the law [12].

In conclusion, the research study that is carried out will be ethical as it can avoid harming human or animal subjects in the sense of knowing which action can be harmful to the research subject.

\section{Ethical Issues of Acupuncture Clinical Research}

Even though many types of research have been done in studying the effect and mechanism of the acupuncture, however, a good design of clinical studies is limited by a lack of information and knowledge regarding the ethical consideration in acupuncture research [13].

Moreover, Miller et al reported [14] that participants usually do not be informed that they may accept a sham acupuncture intervention. They are only be told that there are one or more models of acupuncture being contrast in the trial. Such misleading action lacks persuasive methodological sense and it acts against the ethical requirement of obtaining informed consent with full disclosure. Participants need to be informed with accurate and full disclosure about the use of sham acupuncture. A clinical trial conducted in Germany which is to investigate the effect of acupuncture on migraine by using an acupuncture intervention, a sham acupuncture intervention, and no treatment. Participants were being informed about there are different types of acupuncture intervention that will be taken into comparison. One is alike to the acupuncture intervention used in China while the other is a type that does not follow the principles. However, it has related with the positive result in the trials. Besides, another article used the word 'minimal acupuncture' to described sham acupuncture [15]. It is speculated that this absence of accurate disclosure may be due to the worry of full disclosure would affect the recruitment of participants and diminishment of participant blinding. The patient will feel discomfort with the placebo group and think that sham control is physiologically active. Failure to tell the prospective participant that all of them will have an equal chance to receive a sham or placebo acupuncture treatment can be said as deception. This action does not fulfill the requirement of informed consent but also violates the principle of respect for human beings [16].

Furthermore, there are increasing bioethical issues concerning the method of acupuncture study being carried out. Some people argued that it is unethical to conduct any experiment if the concurrent attempt cannot confirm the treatment plan is better than a placebo or withhold 
treatment for research purposes. This action will delay the recovery period of the patient, while it might also harm the patient [17].

Besides, it comes to a question where the improvement is incompletely due to the efficacy of healing effect of acupuncture, it might be due to chance, acupuncture consultation, patient expectation toward the benefit of acupuncture, putative consequences of appropriate acupuncture needle stimulation at the right acupoints, general needle insertion's physiological effect, selflimited course of the illness, waxing, and waning of the symptoms. Therefore, it is necessary to apply control groups in acupuncture research to examine to what degree the intervention contributes to the sum of therapeutic effects in the patients.

\section{Ways to Conduct Ethical Research}

Although acupuncture treatment has been performed in China for over 3000 years, however, it remains a controversial topic within the medical profession. The rate of production of acupuncture evidence is not satisfactory [18]. Meta-analyses, as well as systematic reviews study about the potential anaesthetic effect of acupuncture treatment haven't been proved to be better than the effect produced by placebo. Many recent systematic reviews have found that the impact of acupuncture on many diseases remains inconclusive. The probable cause, as described in many of the latest Cochrane systematic reviews is due to many clinical trials lacked adequate prescription design and were improperly conducted. Designing high-quality RCTs, scientifically rigorous RCTs of acupuncture remain difficult. This ambiguity generates an ethical dilemma where doctors do not know whether to suggest patients receive acupuncture treatment or other medication because the Good Medical Practice Guidelines (GMC) stated that doctors must perform the most effective treatment according to the best evidence currently available [19].

Standards for Reporting Interventions in Controlled Trials of Acupuncture (STRICA) provide guidelines describing details of apparatus, regime, site, and procedure apply in the study of acupuncture. This checklist is related to the reporting of intervention in the clinical research relating to acupuncture. STRICA is useful in promoting the completeness, clarity, and transparency of the acupuncture protocols and the controls or procedure of comparison in the report of research [20].

"Good research has to be well-calibrated, well planned, properly designed, and ethically validated," says Cope. It underscores the basic requirement of researchers to perform research responsibly. To achieve this, research programs should be developed and followed. It must be carefully agreed upon by all contributors and collaborators, and the exact role of each team member should be clarified early on, including issues of authors and publications. Research should aim to answer specific questions, not just collect data.

Moreover, research involving human beings, medical records, and anonymous human tissues must be approved by an Institutional Review Board or an Ethics Committee. The research proposal needs to discuss the potential ethical issues associated with the research. For the placebocontrolled trials, informed consent should be gained with the information that adequately inform the rationale for the placebo and the danger of it [21].

Before the start of a clinical trial, the study protocol and the necessary documents (case report forms, informed consent, advertisements, and posters) must be submitted to the ethics committee for acceptance. Subjects should be provided with a patient information sheet during the recruitment process, it must contain detailing objectives, procedures, potential benefits and harms, and the right to refuse to participate in the study. Subject or guardian consent should be explained and obtained. Works have to be done to ensure the confidentiality of the information provided by the subject. Schemes and documents can be modified by referencing the comments of reviewers. Clinical trials have to be conducted in accordance with the approved protocol. If there are any modifications, the program will require further approval from the Research Review Board. The intervention plan needs to include acupuncture rationale (such as type of acupuncture used, the principle of acupuncture point selection), acupuncture point selection, needle insertion depth, and needle retention needling details (such as needle length, diameter, and brand name), time, electrical stimulation (frequency and intensity). Details of acupuncture techniques need to be considered, for example, manual manipulation (such as lifting and rotation), or deqi (needle 
sensation). The treatment plan should be clear and specified, such as number of treatments, duration, and frequency of each acupuncture treatment [22]. It should determine the concurrent protocol and use of routine care or ambulance medication during the research. It is important to pay attention to the well-being of the participant. It includes the proper procedure for monitoring of participants which involves precise criteria for early-stage trials termination and standard treatment must be provided [23].

Besides, research needs to be conducted by a qualified acupuncturist. The acupuncturist should have experience in treating the condition being studied. Since acupuncture is 'hands on' type research, thus, the research must be performed by a qualified acupuncturist to avoid the error of practices that might harm the participant. The qualification, clinical experience, and length of training of the acupuncturist must be stated. The effectiveness of acupuncture is influenced by the acupuncturist's experience, for example, the acupuncturist's educational background, years of acupuncture practice, the effectiveness of professional and communication skills. Many adverse events such as cardiac tamponade, organ puncture, and disease transmission are often associated with poorly trained, unlicensed acupuncturists [24].

In the process of planning to publication, researchers are responsible to make sure that research is conducted ethically and responsibly. Researchers should ensure that they are familiar with ethical principles and follow them strictly. Potential ethical issues faced by them in research and publication should be openly discussed within the research team. In case of doubt, it is recommended to consult the expert advice of the relevant Institutional Review Committee (IRB). A clinical researcher must obtain prospective and continuous review by a committee that makes up of members independent of the study to protect the subjects as well as to ensure public liability. Moreover, acupuncture intervention that be compared with biomedical standard care is ethical clinical design as all participant has received treatment. It is best when acupuncture and sham control are compared as adjunctive treatment as all patients can receive standard care. By applying the concept of 'intent to treat', trials that are using an acupuncture group compared with a standard biomedical group or a delayed-treatment group would be expected to be more ethical than the clinical trials using a non-treatment, placebo, or a sham control group [25].

There are seven requirements for ethical research which include social value, fair subject selection, independent review, and respect for the subject, scientific validity, favorable risk-benefit ratio, and informed consent. These requirements put attention on social value. It is important that the clinical research conducted can bring potential clinical significance. If the research lacking value, it is no worth putting the subjects at risk [26]. Moreover, clinical studies need to produce a good riskbenefit ratio that minimizes the risk to the subjects and justifies the risks posed by the potential benefits to the subjects and the value of scientific knowledge gained from the study. Furthermore, the selection of subjects must be consistent with the objectives of the research. The subject should be a competent adult that had been informed about the research details and they must be agreed with it. For incompetent adults and children, the details of the research must be informed to their parents or surrogates and their agreement is required. Besides, researchers have to put consideration to vulnerable groups to avoid ethical violations [27].

\section{Conclusions}

Ethical consideration during acupuncture research is quite important. WHO has stated in one of the documents of 'Guidelines for Clinical Research on Acupuncture, where the concern of human rights should be given to different value systems which deal with human rights such as cultural, social and historical matters? It is the responsibility of researchers to put ethical concerns into consideration in conducting clinical research. Human rights cannot be denied during the research as it is unethical to put the patient health and safety in danger. A well-planned protocol can make sure that the research is carried out systematically while at the same time it is a must to emphasize ethical consideration in the planning stage. 


\section{REFERENCES}

1. Gafarov GA. Acupuncture research methods. J Appl Biotechnol Bioeng 2020;7(6):276-8.

2. Ma Y, Dong M, Zhou K, et al. Publication Trends in Acupuncture Research: A 20-Year Bibliometric Analysis Based on PubMed. PLOS One 2016;11(12):e0168123.

3. Chen H, Yang M, Ning Z, et al. A Guideline for Randomized Controlled Trials of Acupuncture. The American Journal of Chinese Medicine 2019;1-18.

4. Claudia M Witt. Clinical research on acupuncture - Concepts and guideline of efficacy and effectiveness research. Chin J Integr Med. 2011;17(3):166-72.

5. White AR, \& Filshie J, Cummings TM. Clinical trials of acupuncture: consensus recommendations for optimal treatment, sham controls, and blinding. Complement Ther Medicine 2001;9(4):237-45.

6. Lewith G, Field J, Machin D. Acupuncture compared with placebo in post-herpetic pain. Pain. 1983;17:361-8.

7. Lewith GT, Vincent CA. The clinical evaluation of acupuncture. In Filshie J, White A, eds. Medical Acupuncture: a Western Scientific Approach. Edinburgh: Churchill Livingstone, 1998:205-24

8. Miller, FG. Ethical Issues Concerning Research in Complementary and Alternative Medicine. JAMA 2004;291(5):599.

9. Zhong LLD, Wu X, Lam TF, et al. Electro-acupuncture for central obesity: randomized, patientassessor blinded, sham-controlled clinical trial ptorocol. BMC Complement Med Ther 2021;21(1): 190.

10. Jenn NC. Common Ethical Issues In Research And Publication. Malays Fam Physician 2006;1(23):74-6.

11. Zaslawski C. Ethical Considerations for Acupuncture and Chinese Herbal Medicine Clinical Trials: A Cross-Cultural Perspective. Evidence-Based Complementary and Alternative Medicine 2010; 7(3):295-301.

12. Resnik BR. What is Ethics in Research \& Why Is It Important. International Journal of Science and Research (IJSR) 2007;7(10):1299-1300.

13. White A. Editorial Board of Acupuncture in Medicine. Acupunct Med 2009;27(1):33-5.

14. Miller FG, Kaptchuk TJ. Acupuncture trials and informed consent. J Med Ethics 2007;33(1):43-4.

15. Lund I, Haslund J, Lundeberg T. Minimal acupuncture is not a valid placebo control in randomized controlled trials of acupuncture: a physiologist's perspective. Chin Med 2009;4:1.

16. Department of Health, Education, and Welfare; National Commission for the Protection of Human Subjects of Biomedical and Behavioral Research. The Belmont Report. Ethical principles and guidelines for the protection of human subjects of research. J Am Coll Dent 2014;81(3):4-13.

17. MacPherson $\mathrm{H}$, Altman DG, Hammerschlag $\mathrm{R}$, et al. Revised STandards for Reporting Interventions in Clinical Trials of Acupuncture (STRICTA): Extending the CONSORT Statement. PLoS Med 2010;7(6):e1000261.

18. Connelly P. The Ethics of Acupuncture. Scottish Universities Medical Journal 2012;1(2):165-9.

19. Lyons $\mathrm{D}$, Lyall $\mathrm{D}$. Developing good practice guidelines for the administration of covert medication. BMC Psychiatry, 2007;7:S41.

20. MacPherson H, Altman DG, Hammerschlag R, et al. Revised Standards for Reporting Interventions in Clinical Trials of Acupuncture (STRICTA): extending the CONSORT statement. PLoS Med 2010;7(6):e1000261.

21. Suarez-Almazor ME, Looney C, Liu Y, et al. A randomized controlled trial of acupuncture for osteoarthritis of the knee: effects of patient-provider communication. Arthritis Care Res (Hoboken) 2010;62(9):1229-36.

22. Hu NJ, Liu YQ, Zhao MY, et al. Influence of the Intensity, Components, and Spreading of the Deqi Sensation on the Analgesic Effect of SP6 Needling in Primary Dysmenorrhea Patients: A Secondary Analysis of a Randomised Controlled Trial. Evid Based Complement Alternat Med 2019:6462576.

23. Lewith GT, White PJ, Kaptchuk TJ. Developing a research strategy for acupuncture. Clin J Pain 2006;22(7):632-8.

24. Birch S, Hesselink JK, Jonkman FAM, et al. Clinical Research on Acupuncture: Part 1. What Have Reviews of the Efficacy and Safety of Acupuncture Told Us So Far? J Alternat Complement Med 2004;10(3):468-80.

25. Hammerschlag R. Methodological and Ethical Issues in Clinical Trials of Acupuncture. J Alternat Complement Med 1998;4(2):159-71.

26. Ezekiel JE, David W, Jack K, et al. What Makes Clinical Research in Developing Countries Ethical? The Benchmarks of Ethical Research. J Infectious Dis 2004;5(1):930-7. 
27. Han JS, Ho YS. Global trends and performances of acupuncture research. Neurosci Biobehav Rev 2011;35(3):680-7.

$* * * * * * * * * * * * * * * * * * * * * * * * * * * * * * * *$

Acknowledgements: Nil

Conflict of interest: Nil

Funding: Nil 